\title{
O Uso da Acupuntura na Sintomatologia do Stress
}

Acupuncture Effectiveness for Stress Symptoms

El Uso de la Acupuntura En La Sintomatología Del Stress

Marília Conceição da Silva Doria, Marilda Emmanuel Novaes Lipp Pontifícia Universidade Católica de Campinas

Delvo Ferraz da Silva Instituto de Psicologia e Acupuntura - Espaço

Consciência
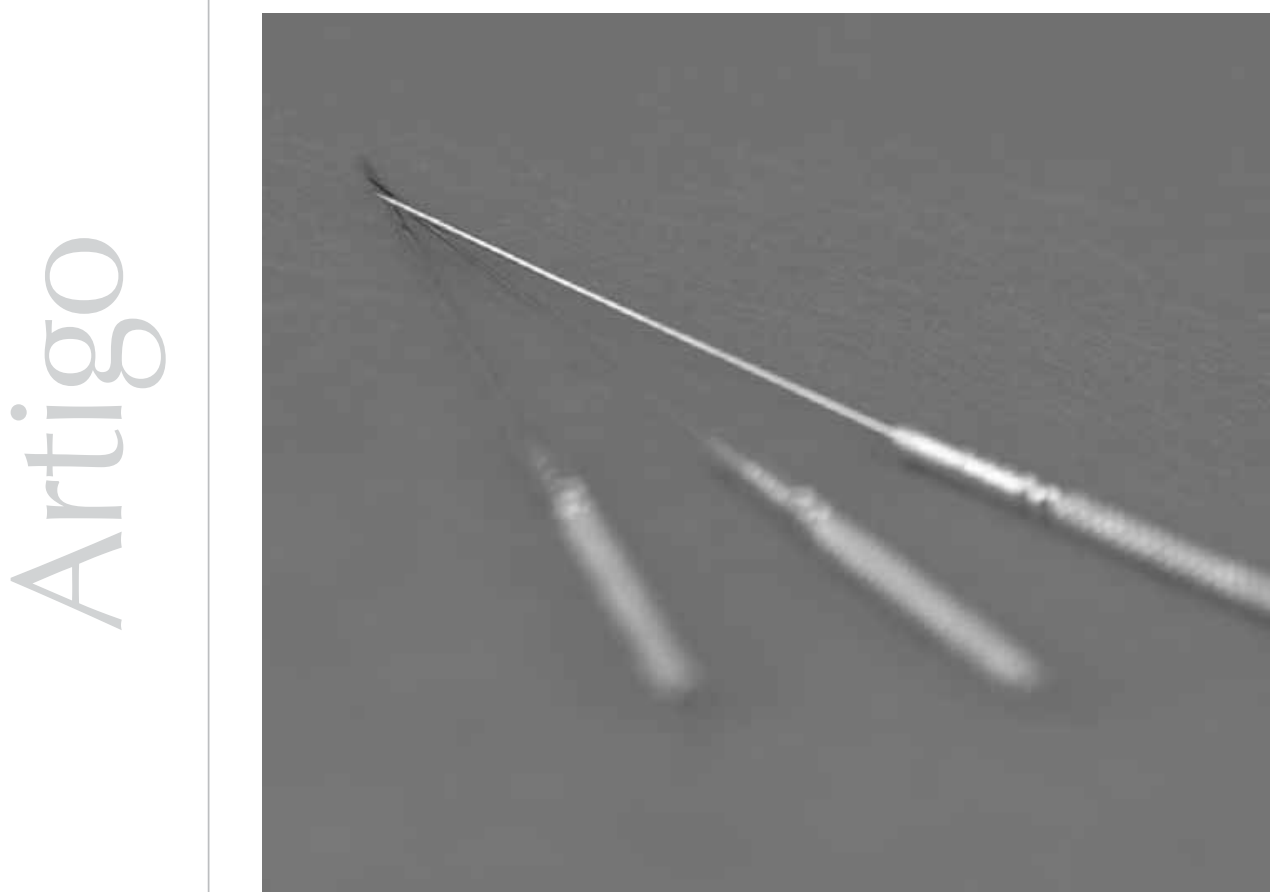
Resumo: O presente estudo objetivou verificar o uso da acupuntura na sintomatologia do stress. Os participantes foram 20 adultos, 15 mulheres e 5 homens, na faixa etária de 27 a 65 anos. Todos assinaram o Termo de Consentimento e foram submetidos à aplicação do Inventário de Sintomas de Stress de Lipp, para avaliar a presença de stress, a um questionário e à Escala Analógica Visual, para avaliar a intensidade da queixa. Assim, os participantes que atingiram os critérios de inclusão participaram do tratamento de acupuntura, que foi realizado em 10 sessões individuais. Após as 10 sessões, os participantes foram reavaliados com a EAV e o ISSL. Os resultados gerais mostraram que antes do tratamento de acupuntura, $100 \%$ dos participantes apresentavam stress, sendo que $60 \%$ se encontravam na fase de resistência, e a média da intensidade da queixa era 8,1, em uma escala que variava de 1 a 10 . O tratamento foi capaz de reduzir significativamente a presença de stress $(p<0,001)$, o nível de stress $(p=0,006)$, a predominância de sintomas $(p=0,02)$ e a intensidade da queixa $(p<0,001)$. Conclui-se que o tratamento de acupuntura foi útil na redução da sintomatologia do stress dessa amostra, considerando-se seus efeitos imediatos.

Palavras-chave: Stress. Medicina tradicional chinesa. Terapia multimodal. Acupuntura. Psicologia.

Abstract: The present study aimed to verify the acupuncture effectiveness for stress symptoms treatment. The 20 participants were all volunteers from the community, 15 female and 5 male, aged from 27 to 65 years old. The Free and Informed Consent was signed, and then the application of the Lipp Stress Symptoms Inventory, an interview and the Analogue-Visual Scale, to evaluate the participants' demand. The participants' who attended the inclusion criterion had received 10 acupunture sessions. All participants were submitted to the EAV e ISSL after the 10 sessions. The general results indicated that before the acupuncture treatment $60 \%$ of the volunteers were in the resistance phase of stress, and the demand intensity average was 8,1 , considering a scale from 1 to 10 . After the treatment, the stress presence was significantly reduced $(p<0,001)$, as the stress level $(p=0,006)$, the symptoms predominance $(p=0,02)$, and the demand intensity $(p<0,001)$. As the acupuncture treatment reduced significantly the stress symptoms presence, it's possible to conclude its effectiveness in this sample.

Keywords: Stress. Medicine chinese tradiotional. Multidmodal treatment approach. Acupuncture. Psychology.

Resumen: El presente estudio objetivó verificar el uso de la Acupuntura en la sintomatología del stress. Los participantes fueron 20 adultos, siendo 15 mujeres y 5 hombres, en la franja etaria de 27 a 65 años. Los participantes firmaron el Término de Consentimiento, y fueron sometidos a la aplicación del Inventario de Síntomas de Stress de Lipp, para evaluar la presencia de stress, a un cuestionario, y a la Escala Analógica Visual, para evaluar la intensidad de la queja. Así, los participantes que alcanzaron los criterios de inclusión, participaron en el Tratamiento de Acupuntura, que fue realizado en 10 sesiones individuales. Después de las 10 sesiones, los participantes fueron reevaluados con la EAV y el ISSL. Los resultados generales mostraron que antes del Tratamiento de Acupuntura, el 100\% de los participantes presentaban stress, siendo que el 60\% se encontraban en la fase de resistencia, y la media de la intensidad de la queja era 8,1 , en una escala que variaba de 1 a 10. El tratamiento fue capaz de reducir significativamente la presencia de stress $(p<0,001)$, el nivel de stress $(p=0,006)$, la predominancia de síntomas $(p=0,02)$ y la intensidad de la queja $(p<0,001)$. Se concluye que el Tratamiento de Acupuntura fue útil en la reducción de la sintomatología del stress de esta muestra, considerándose sus efectos inmediatos.

Palabras clave: Stress. Medicina tradicional chinesa.Terapia multimodal. Acupuntura. Psicología.

A presente pesquisa caracteriza-se como um estudo preliminar que teve por objetivo expandir as terapêuticas e os programas voltados para o controle do stress, com o intuito de garantir a saúde e o bem-estar de pacientes estressados. Sendo assim, esta pesquisa enfatizou o uso da acupuntura na sintomatologia do stress.

Para Lipp (1996), o stress é definido como uma reação do organismo, possui componentes físicos e/ou psicológicos, e é causado pelas alterações psicofisiológicas que ocorrem quando a pessoa se confronta com uma determinada situação, seja ela amedrontadora, seja ela feliz. Na realidade, o stress é um estado de tensão mental e físico que produz um desequilíbrio no funcionamento global do ser humano e enfraquece seu sistema imunológico, deixando-o sujeito a infecções e a doenças.

O stress, segundo Selye (1965), desenvolve-se em três fases principais:

Fase de alerta: é a primeira fase, que ocorre 
quando o indivíduo percebe o agente estressor ou se vê exposto a ele. A partir daí, o organismo prepara-se para lutar ou fugir, quando ocorre grande atividade do sistema nervoso simpático e quando alterações hormonais são segregadas pela glândula suprarrenal (adrenalina e noradrenalina). Caso o estressor tenha uma duração curta, essa fase termina após algumas horas, quando se elimina a adrenalina produzida, e a restauração da homeostase ocorre sem nenhum dano ao organismo.

Algumas atividades de baixa prioridade são suspensas, como comer e digerir alimentos, para armazenar energia. Dessa maneira, o organismo utiliza-se de todas essas fontes quando ameaçado por um estressor, seja para mobilizar-se e atacar o inimigo, seja para escapar em segurança (Selye, 1965).

Lipp (1984) coloca como sintomas característicos dessa fase: aumento da frequência cardíaca, esfriamento de mãos e pés, insônia, taquicardia, hiperventilação, aumento da sudorese, mudança de apetite, tensão muscular, entusiasmo súbito e aumento súbito de motivação. É nessa fase que a produtividade aumenta a ponto de, sabendo lidar e administrar o stress, a pessoa poder utilizá-lo a seu favor, pois a motivação, o entusiasmo e a energia que esse estágio produz podem favorecer a produtividade.

Se o estressor permanecer por um período prolongado ou se for muito intenso, mesmo que em um período curto, o organismo passa para a fase de resistência.

Fase de resistência: é a segunda fase do stress, que ocorre quando o estressor perdura por um período muito prolongado. Nessa fase, o organismo tenta adaptar-se ou mesmo, como o próprio nome sugere, tenta resistir ao que está vivenciando, e utiliza suas reservas de energia adaptativa para o reequilíbrio.

Holmes e Rahe (1967) enfatizam o conceito de energia adaptativa, conforme o qual cada pessoa possui uma quantidade fixa de energia, mas, sendo esta renovável, é usada para as mudanças significativas que ocorrem em sua vida.

Esses autores relacionaram problemas de saúde a mudanças significativas na vida de uma pessoa (morte de cônjuge, mudança de emprego), observando que, quanto maior o número de mudanças ocorridas em sua vida no espaço de um ano, mais energia ela utiliza - e, portanto, menor energia adaptativa possui naquele momento para enfrentar estressores. Desse modo, torna-se maior a probabilidade de ocorrerem problemas de saúde.

Nessa fase, dois sintomas são frequentes: sensação de desgaste físico generalizado, sem causa aparente, e dificuldades com a memória. No nível fisiológico, ocorrem alterações no funcionamento das glândulas suprarrenais. A medula diminui a produção de adrenalina e o córtex produz mais corticoides. Entretanto, se o estressor permanecer por muito tempo, as reservas de energia adaptativa também cessarão, e o processo de stress progredirá para a fase de exaustão. No final da fase de resistência, o organismo enfraquece e tornase vulnerável a várias doenças, como herpes simples, psoríase, aumento da pressão e desencadeamento da diabetes em indivíduos com predisposição genética. Algumas pessoas podem também desenvolver retração de gengiva, gripe, tontura, sensação de levitação e redução da libido (Lipp \& Malagris, 1995). Caso a pessoa não consiga sair dessa fase e nela permaneça por um tempo considerável, o processo de stress pode desenvolver-se até o ponto mais crítico, que é a fase de exaustão.

Fase de exaustão: é a terceira fase do stress. Nessa fase, o stress torna-se intenso e, em consequência, faz esgotar a energia adaptativa do organismo.

Segundo Lipp e Rocha (1996), dificilmente se consegue sair do estágio de exaustão sem 
Como forma

de controle do stress, Lipp,

Malagris e Novais (2007) apontam

quatro pilares:

alimentação

saudável e

equilibrada,

relaxamento,

prática regular e

sistemática de

exercícios físicos

e reestruturação cognitiva,

que parte da

hipótese de que

pensamentos geram

sentimentos,

e assim os

pensamentos

distorcidos e

negativos devem

ser substituídos

por pensamentos

mais funcionais

para o bem-estar

do indivíduo. tratamento especializado, que combina intervenção médica e psicológica. As reações tornam-se altamente nocivas, o organismo torna-se vulnerável a doenças e disfunções, podendo resultar em morte (Spielberger, 1979). Com base nas três fases conceituadas por Selye (1965), Lipp (2000) sugeriu um modelo quadrifásico do stress, que inclui uma fase adicional entre a resistência e a exaustão chamada de quase-exaustão. Essa fase caracteriza-se por um enfraquecimento da pessoa, que não mais está conseguindo se adaptar ou resistir ao estressor. As doenças começam a surgir, porém ainda não são tão graves como na fase de exaustão. Embora apresentando desgaste e outros sintomas, a pessoa ainda consegue, até certo tempo, trabalhar e produzir, ao contrário do que ocorre na fase de exaustão, quando o indivíduo para de produzir adequadamente, não conseguindo, na maioria das vezes, trabalhar nem se concentrar.

Como forma de controle do stress, Lipp, Malagris e Novais (2007) apontam quatro pilares: alimentação saudável e equilibrada, relaxamento, prática regular e sistemática de exercícios físicos e reestruturação cognitiva, que parte da hipótese de que pensamentos geram sentimentos, e assim os pensamentos distorcidos e negativos devem ser substituídos por pensamentos mais funcionais para o bemestar do indivíduo.

Lipp, Malagris e Novais (2007) finalizam sua reflexão observando que existem muitos métodos de controle de stress, a maioria dos quais objetiva reduzir a tensão física e mental, como massagem, yoga e acupuntura. Assim sendo, focou-se neste último como um método de controle do stress.

\section{O stress na visão da Medicina Tradicional Chinesa - MTC}

Na medicina tradicional chinesa, não se trata uma doença, e, sim, o padrão energético desarmônico que a causa. Entende-se a saúde como resultado do equilíbrio entre Yin e Yang. Quando estes estão em desequilíbrio, ocorre o processo de adoecimento. A doença não surge de uma hora para outra, mas é fruto de uma sucessão de experiências estressantes, acompanhadas por uma fragilidade do mecanismo de proteção (Maciocia, 1996).

A medicina tradicional chinesa (MTC) entende que o comportamento do homem não é determinado apenas pelo cérebro, mas que o indivíduo é um microcosmo inserido no macrocosmo (universo), e que este influencia e é influenciado por ele (Breves, 2001).

Faubert e Crepon enfatizam:

Segundo a tradição chinesa, o ser humano constitui uma só entidade energética, e não é suscetível de ser dividido. O psiquismo não pode, portanto, em caso algum, ser dissociado do físico: ambos apresentam manifestações diferentes da mesma energia, eles seguem as mesmas leis e estão em interdependência completa, como as duas faces da mesma folha de papel. No caso de perturbações, seja do psiquismo ou do organismo, não se poderia, em absoluto, tratar de um sem referência ao outro (1990, p. 95)

A base de tudo é o Qi, a força vital. Mas não se trata apenas da força vital do ser humano ou do animal, sendo essa uma energia universal que cerca e permeia todas as coisas, animadas e inanimadas, e que circula no organismo por canais denominados meridianos. Os chineses, portanto, encaram todo o funcionamento do organismo e da mente como dependente do fluxo normal das energias do organismo ou da força vital, a que denominamos Qi (Kidson, 2006).

A acupuntura é uma das técnicas da MTC, e é baseada nas teorias Yin e Yang e dos cinco elementos.

O conceito de Yin/Yang, juntamente ao do Qi, tem permeado a filosofia chinesa há séculos. 
Yin e Yang representam não só qualidades opostas, mas também complementares. Cada coisa ou fenômeno pode existir por si mesma ou pelo seu oposto. Além disso, Yin contém a semente de Yang e vice-versa. A partir desse ponto de vista, Yin e Yang são dois estágios de um movimento cíclico, sendo que um interfere constantemente no outro, tal como o dia cede lugar à noite $\mathrm{e}$ vice-versa (Maciocia, 1996).

O corpo humano é um todo organizado, composto de duas partes ligadas estruturalmente, porém opostas; Yin/Yang são os dois polos que estabelecem os limites para os ciclos de mudanças (Wen, 1989).

A medicina tradicional chinesa baseia-se no equilíbrio dessas duas forças no corpo humano, sendo a doença vista como um rompimento desse equilíbrio. As duas partes do corpo, Yin/Yang, devem estar em equilíbrio relativo para que se mantenham normais as suas atividades fisiológicas. O equilíbrio é destruído por fatores de adoecimento, podendo ocorrer o predomínio ou a falta de uma das duas partes, vindo a ocorrer processos patológicos (Wen, 1989).

A outra base primordial da medicina tradicional chinesa é a teoria dos cinco elementos, que se caracteriza pelo ciclo de geração e de controle. No ciclo de geração, os elementos sucedemse ininterruptamente, cada um tendo origem no que lhe antecede e dando origem ao que lhe sucede. Assim, o Fogo dá origem à Terra (as cinzas); a Terra dá origem ao Metal (porque o contém); o Metal dá origem à Água (porque se liquefaz); a Água dá origem à Madeira (porque nutre o vegetal), e a Madeira dá origem ao Fogo (porque é combustível). Já no ciclo de controle, cada elemento inibe aquele que sucede o elemento gerado. Assim, o Fogo funde o Metal, o Metal corta a Madeira, a Madeira cobre a Terra, a Terra absorve a Água e a Água extingue o Fogo. Os ciclos estão ilustrados na Figura 1 (Cordeiro \& Cordeiro, 2001).

Segundo Tymowski, Guillaume e Fiévet-Izard (1986), para que esse conjunto fique perfeitamente equilibrado e o ciclo gire corretamente, deve-se obedecer aos dois ciclos.

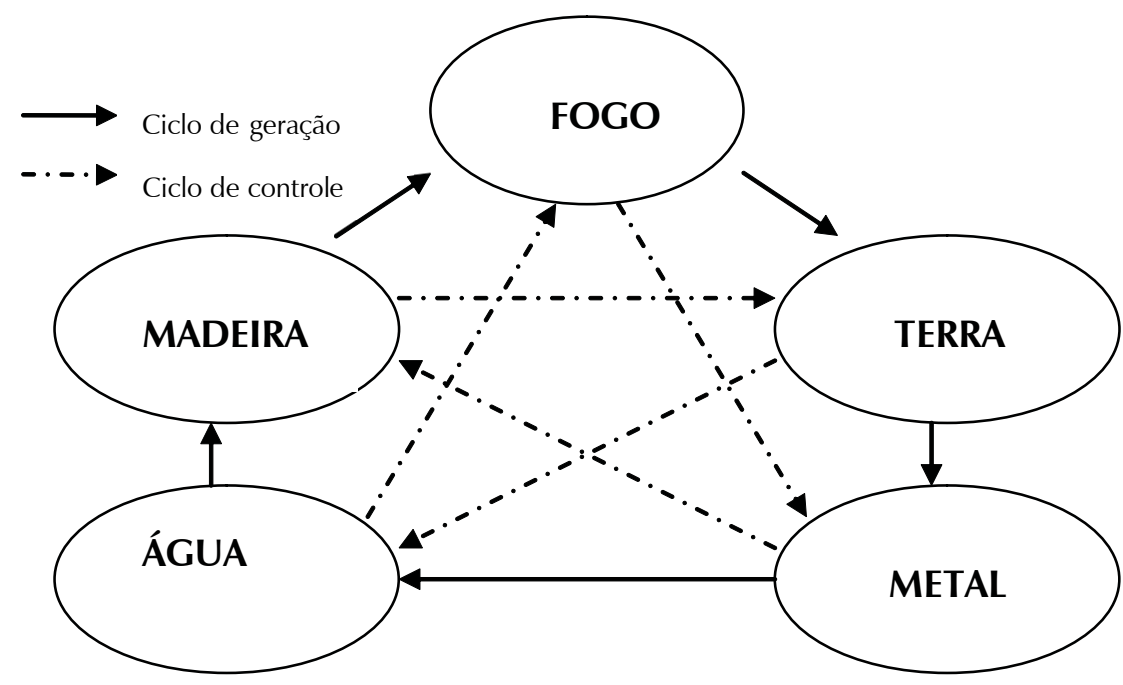

Figura 1. Diagrama dos cinco elementos

Fonte: Cordeiro \& Cordeiro (2001) 
As relações entre os cinco elementos e os constituintes do organismo, descritos por Maciocia, estão ilustradas na Tabela 1.

Tabela 1. Correspondência dos cinco elementos

\begin{tabular}{llllll}
\hline Cinco elementos & Madeira & Fogo & Terra & Metal & Água \\
\hline $\begin{array}{l}\text { Órgão (Zang) } \\
\text { Víscera (Fu) }\end{array}$ & $\begin{array}{l}\text { Fígado } \\
\text { Vesícula } \\
\text { biliar }\end{array}$ & $\begin{array}{l}\text { Coração } \\
\text { Intestino } \\
\text { delgado }\end{array}$ & $\begin{array}{l}\text { Baço-pâncreas } \\
\text { Estômago }\end{array}$ & $\begin{array}{l}\text { Pulmão } \\
\text { Intestino } \\
\text { grosso }\end{array}$ & $\begin{array}{l}\text { Rins } \\
\text { Banifestação } \\
\text { externa }\end{array}$ \\
$\begin{array}{l}\text { Manifestação } \\
\text { energética }\end{array}$ & Olho & Língua & Boca & Nariz & Orelha \\
$\begin{array}{l}\text { Energia } \\
\text { Cor }\end{array}$ & Unha & Tez do rosto & Lábios & Pele/pelos & Cabelo \\
Emoções & Vento & $\begin{array}{l}\text { Calor } \\
\text { Vermelho }\end{array}$ & $\begin{array}{l}\text { Umidade } \\
\text { Amarelo }\end{array}$ & $\begin{array}{l}\text { Secura } \\
\text { Branco }\end{array}$ & $\begin{array}{l}\text { Frio } \\
\text { Preto }\end{array}$ \\
\hline
\end{tabular}

Deve-se avaliar o organismo humano tomando como referência os ciclos (de geração e de controle) dos cinco elementos, para identificar os desequilíbrios e orientar a intervenção no sentido de restaurar as leis do sistema.

Cordeiro (1994) explica que o pensamento chinês não separa o corpo da mente, mas considera o indivíduo como um todo, de maneira global, e, por isso, as características psíquicas de cada um orientam o terapeuta para o diagnóstico concomitante do estado físico e psíquico do paciente. Em consequência, a ação da acupuntura, no corpo físico, repercute inevitavelmente no estado psíquico.

O diagnóstico mais importante para o tratamento com acupuntura é o diagnóstico etiológico, pois procura buscar a causa da patologia e do desequilíbrio energético, trata dos fatores que determinam o aparecimento das patologias no homem e, por meio da anamnese, verifica a história clínica do paciente, com base nos sinais e sintomas e também nas suas condições de vida, como a prática de exercício físico, a alimentação equilibrada, a observação da cor da face e a avaliação dos microssistemas: pulso, língua e orelha, entre outros, já que o microssistema, por exemplo, a orelha, é uma parte do organismo que caracteriza o organismo como um todo (macrossistema).

Os relacionamentos de geração e controle mútuo entre os elementos são um bom modelo de alguns processos autorreguladores de equilíbrio que podem ser encontrados na Natureza e no organismo (Maciocia, 1996). Dessa forma, para a medicina tradicional chinesa, o stress é considerado um desequilíbrio energético do Yin e Yang, e, assim, sugere-se que os ciclos de geração e inibição da lei dos cinco elementos possam ser utilizados no seu tratamento.

Porém, como cada indivíduo diagnosticado com stress pode apresentar sintomas diferentes dos apresentados por outrem, ou seja, como o desequilíbrio energético é individualizado, o tratamento de acupuntura deve também ser realizado de forma individualizada, de acordo com o desequilíbrio de cada paciente.

Um paciente diagnosticado com stress que apresente medo, por exemplo, revela que há um desequilíbrio no elemento Água. O tratamento de acupuntura parte da hipótese de que, no 
meridiano do rim ou da bexiga, pertencente ao elemento Água, há um desequilíbrio energético. Assim, podemos verificar no microssistema pulso se a energia desses meridianos está em excesso ou é insuficiente. Se houver excesso, a energia deve ser sedada, mediante a aplicação de agulha no ponto de sedação do meridiano. Se houver insuficiência, a energia deve ser tonificada, mediante a aplicação de agulha no ponto de tonificação do meridiano (seguindo a teoria dos cinco elementos).

Requena (1990) enfatiza que, para a MTC, a constituição genética de cada indivíduo irá definir a vulnerabilidade específica de um ou outro órgão, considerado este como órgãoalvo a ser desequilibrado primeiramente no momento de stress.

Com base na especificação de sintomas constantes no ISSL (Inventário de Sintomas de Stress de Lipp, 2000), e de acordo com as orientações do psicólogo acupunturista Delvo Ferraz da Silva, propõe-se que, para o tratamento de stress com acupuntura, sejam definidos seis tipos de desequilíbrio causados pelo stress, de acordo com a teoria dos cinco elementos:

Tipo 1 - Elemento Metal $\rightarrow \quad$ Campiglia (2004) afirma que o elemento Metal, na MTC, representa o órgão pulmão e a víscera intestino grosso. Por isso, todos os padrões de adoecimento relacionados ao elemento Metal são considerados como desarmonias do sistema pulmão ou do sistema intestino grosso; assim, associa-se ao elemento Metal o controle da respiração. Esta representa a vida e a morte, a possibilidade de entrar o novo e sair o velho, o desapego, o incessante movimento da vida, a impermanência. $\mathrm{O}$ elemento Metal exterioriza-se pelo nariz, manifesta-se na pele e nos pelos, regula a abertura e o fechamento dos poros e controla a tristeza. Ele está relacionado com a energia secura e contém o Pro, uma entidade visceral que dá ao corpo a capacidade de coordenação motora, de equilíbrio, de agilidade física e envolve todos os reflexos, os impulsos e as sensações corporais.

Sintomas mencionados no ISSL: hiperventilação (a respiração está relacionada ao sistema pulmão), problemas dermatológicos (o sistema pulmão é o responsável pela pele), angústia/ansiedade diária.

Tipo 2 - Elemento Água $\rightarrow$ Segundo Campiglia, o elemento Água, na MTC, representa o órgão rim e a víscera bexiga. Por isso, todos os padrões de adoecimento relacionados ao elemento Água são considerados como desarmonias do sistema rim ou do sistema bexiga. Assim, associamse ao elemento Água: os ouvidos, o cérebro, a medula, os ossos, os dentes, a região lombar, o aparelho reprodutor, o controle do fluxo dos líquidos corporais, os cabelos (que mostram em que estado se encontra a energia do sistema rim), o medo, o espírito de decisão, que está relacionado com a energia frio e a entidade visceral Tche, à qual está relacionada a tenacidade, a coragem, o espírito de decisão, a determinação, a execução de ideias, o interesse, a ambição, a adaptação presente em cada indivíduo e a força de vontade.

Sintomas mencionados no ISSL: impossibilidade de trabalhar e sensação de incompetência em todas as áreas (devido à falta de energia armazenada pelo sistema rim e à insegurança do indivíduo, que é controlada pelo sistema rim), hipertensão arterial (devido à deficiência do sistema rim, o elemento Água não controla o elemento Fogo, pelo ciclo de inibição).

Tipo 3 - Elemento Madeira $\rightarrow$ Campiglia afirma que o elemento Madeira, na MTC, representa o órgão fígado e a víscera vesícula biliar. Por isso, todos os padrões de adoecimento relacionados ao elemento 
Madeira são considerados como desarmonias do sistema fígado ou do sistema vesícula biliar. Assim, associam-se ao elemento Madeira: o controle dos sentimentos, a regulação da digestão e a realização do movimento de recolhimento do sangue no corpo durante o sono. Isso faz com que os batimentos cardíacos e a respiração diminuam e o indivíduo durma. O elemento Madeira é o símbolo do crescimento. Está ligado à raiva e à energia vento, expressa-se pelos olhos, está associada aos tendões, aos ligamentos e às unhas, e contém a entidade visceral Hun, que representa a figura do general e que é responsável pela inteligência instintiva, não sendo guiado pela razão, como o Shen.

Sintomas mencionados no ISSL: insônia (o sistema fígado é responsável por recolher o sangue no momento do sono, diminuindo a frequência cardíaca e respiratória), tontura (o excesso da energia vento provoca a tontura), sensibilidade emotiva excessiva (as emoções são coordenadas pelo Hun), irritabilidade excessiva, aperto da mandíbula/ranger de dentes, tiques (movimento do vento, que é controlado pelo sistema fígado), excesso de gases (movimento do vento, que é controlado pelo sistema fígado), raiva, pesadelos (agitação causada pela energia vento).

Tipo 4 - Elemento Fogo $\rightarrow$ Para Campiglia (2004), o elemento Fogo, na MTC, representa o órgão coração e a víscera intestino delgado. Por isso, todos os padrões de adoecimento relacionados ao elemento Fogo são considerados como desarmonias do sistema coração ou do sistema intestino delgado. O elemento Fogo controla o suor e exterioriza-se pela língua, razão pela qual a fala também é uma manifestação desse elemento. A ele associa-se a alegria. O elemento Fogo simboliza o calor e contém a entidade visceral Shen, que pode ser observada na tez e na expressão do rosto, está associada à inteligência, à sabedoria, à razão guiada pelos princípios e pela moral, à consciência de quem somos, das nossas potencialidades e à consciência que desenvolvemos com nossas experiências de vida.

Sintomas mencionados no ISSL: perda do senso de humor (a alegria é controlada pelo sistema coração), infarto (deficiência no sistema coração).

Tipo 5 - Elemento Terra $\rightarrow$ Segundo Campiglia, o elemento Terra, na MTC, representa os órgãos baço-pâncreas e a víscera estômago. Por isso, todos os padrões de adoecimento relacionados ao elemento Terra são considerados como desarmonias do sistema baço-pâncreas ou do sistema estômago. Assim, associam-se ao elemento Terra: o transporte e a transformação de energia provenientes dos alimentos, a contenção do sangue nos vasos e o comando dos vasos e do sangue (mestre do sangue). Esse elemento cuida dos quatro membros, manifesta-se nos lábios e exterioriza-se pela boca. Ele controla o pensamento e o raciocínio, é responsável pelo tônus muscular, está relacionado com a energia umidade e contém a entidade visceral I, que corresponde à possibilidade de imaginação, ideação, reflexão, desejo, concentração, memorização e compreensão.

Sintomas mencionados no ISSL: diarreia, nó no estômago, mudança de apetite, malestar generalizado sem causa específica, náusea, aparecimento de úlcera (sintomas relacionados à transformação dos alimentos, função do sistema BP e do sistema E), pensar constantemente em um só assunto (controla os pensamentos) e apatia.

Tipo 6 - Elementos combinados $\rightarrow$ Ocorre a combinação quando há desequilíbrio em mais de um elemento.

Sintomas mencionados no ISSL: boca seca (elemento Terra/ sistema baço-pâncreas: 
exterioriza-se na boca; elemento Metal/ sistema pulmão: controla a secura), sensação de desgaste físico e cansaço constantes (elemento Terra/ sistema baço-pâncreas: cuida dos quatro membros; elemento Água/ sistema rim: comanda as energias do corpo), aumento da sudorese (elemento Metal/ sistema pulmão: pele; elemento Água: suor que escorre; elemento Terra: umidade, suor que gruda; elemento Fogo: excesso de calor provoca o suor; elemento Madeira: o excesso de vento faz o suor sair); tensão muscular (elemento Madeira/ sistema fígado-vesícula biliar: controla a tensão; elemento Terra/ sistema baço-pâncreas: controla os músculos), aumento súbito de motivação e entusiasmo súbito (elemento Água e elemento Terra são responsáveis pela produção de líquidos e hormônios do corpo), vontade súbita de iniciar novos projetos e vontade de fugir de tudo (elemento Água/ sistema rim: controla a força de vontade; elemento Madeira/ sistema fígado: comanda a ação), problemas com a memória (elemento Terra/ sistema baço-pâncreas: responsável pela concentração e memorização nos estudos; elemento Fogo/ sistema coração: responsável pela memória remota; elemento Água/ sistema rim: responsável pela memória recente), mãos e pés frios, formigamento das extremidades (elemento Terra/ sistema baço-pâncreas: cuida dos quatro membros; elemento Água/ sistema rim: controla o frio, cujo excesso é a causa do formigamento), diminuição da libido (elemento Água/ sistema rim: responsável pela vontade; elemento Fogo/ sistema coração: relacionado ao sistema circulação-sexualidade), dúvida quanto a si próprio (elemento Água/ sistema rim: relaciona-se à insegurança e à dúvida; elemento Fogo/ sistema coração (Shen): responsável pela consciência de quem somos), falar/pensar constantemente em um só assunto (elemento Fogo/ sistema coração: responsável pela fala; elemento Terra: pensamento e repetição de um assunto está relacionado ao I).
A partir dessa revisão bibliográfica, a presente pesquisa caracteriza-se como um estudo preliminar que poderá trazer benefícios no sentido de expandir as terapêuticas e programas voltados para o controle do stress, com o intuito de garantir a saúde e o bem-estar de pacientes estressados. Nesse sentido, o objetivo principal deste trabalho foi pesquisar o uso da acupuntura na sintomatologia do stress.

\section{Método}

A amostra foi composta por 20 adultos, 15 mulheres e 5 homens, na faixa etária de 27 a 65 anos, provenientes da comunidade em geral, os quais responderam ao anúncio da pesquisadora para participação na pesquisa, sendo que $65 \%$ da amostra possuía ensino superior completo e $65 \%$ eram solteiros.

Os critérios para inclusão da amostra foram: a) pertencer à faixa etária de 21 a 65 anos, já que a demanda pelo tratamento por acupuntura é maior nessa faixa etária; b) possuir uma queixa que se caracterizasse como um dos sintomas de stress que consta no ISSL; c) ter sido diagnosticado com stress; d) ter nível de escolaridade acima do ensino fundamental, a fim de garantir a compreensão dos instrumentos utilizados para a realização da pesquisa; e) não possuir distúrbio psiquiátrico grave previamente diagnosticado; f) ter disponibilidade de tempo para participar das avaliações no início e no fim do estudo, bem como do tratamento das 10 sessões de acupuntura e g) aceitar participar da pesquisa.

Os materiais utilizados foram: a) Termo de Consentimento Livre e Esclarecido; b) questionário elaborado especificamente para esta pesquisa, composto de questões abertas, que tinha o objetivo de obter dados de identificação e dados clínicos, como queixa (sintoma principal do ISSL), tempo da queixa e outras queixas); c) Escala 
Analógica Visual - EAV, a intensidade da queixa (sintoma principal do ISSL) do participante, que foi avaliada de acordo com a Escala Analógica Visual, em uma gradação de 1 a 10, mediante marcação, feita pelo participante, no ponto subjetivamente correspondente à intensidade de sua queixa. Trata-se de uma linha horizontal ou vertical de 10 centímetros, em cujos extremos aparecem às designações pouco incômodo (1) e muito incômodo (10). Na utilização dessa escala, solicita-se ao participante que marque livremente um ponto que indique sua autoavaliação sobre a queixa experimentada; d) Inventário de Sintomas de Stress para Adulto - ISSL (Lipp, 2000), para avaliar a presença de stress; e) cartaz de divulgação da pesquisa para psicólogos acupunturistas, que foi enviado por e-mail para psicólogos acupunturistas formados pelo Instituto de Psicologia e Acupuntura - Espaço Consciência, de São Paulo, com o objetivo de recrutá-los para realizar o tratamento de acupuntura da pesquisa; f) cartaz de divulgação da pesquisa para os participantes, os quais foram fixados em locais públicos da cidade de São Paulo, convidando as pessoas a participarem da pesquisa; g) folha de registro para o tratamento por acupuntura, que é a folha em que se registram as anotações gerais sobre o tratamento de acupuntura de cada participante. Os itens que constituem a folha de registro são: iniciais, $\mathrm{n}$ o do prontuário, data de início e de término do tratamento, princípio terapêutico, proposta terapêutica, procedimento terapêutico geral de cada sessão, conclusão; h) questionário de avaliação e procedimentos para o tratamento por acupuntura, que trata da avaliação das queixas, e da avaliação dos três microssistemas: orelha, língua e pulso, realizadas a cada sessão, antes de o participante ser submetido à aplicação de agulhas e outros procedimentos da MTC; i) orientações sistematizadas sobre o tratamento de acupuntura na sintomatologia do stress, propostas pelo professor Delvo
Ferraz da Silva, que foram passadas aos psicólogos acupunturistas.

O questionário e as avaliações psicológicas, assim como o tratamento de acupuntura, foram realizados em salas para atendimento individual, dotadas dos recursos necessários para o tratamento, localizadas no Instituto de Psicologia e Acupuntura - Espaço Consciência, na cidade de São Paulo, com autorização do coordenador.

Duas mestrandas colaboraram com esta pesquisa, aplicando o questionário e as avaliações psicológicas (ISSL e EAV) antes e depois do tratamento de acupuntura, com a pesquisadora. Embora sendo psicóloga acupunturista, a pesquisadora optou em não aplicar ela própria o tratamento de acupuntura, para evitar vieses e fornecer uma visão mais neutra na pesquisa. Assim sendo, o tratamento foi realizado por uma equipe de cinco psicólogos acupunturistas, com orientações sistematizadas e sob supervisão do professor Delvo Ferraz da Silva. Cada psicólogo acupunturista ficou responsável pelo tratamento de quatro participantes.

\section{Procedimento}

Inicialmente, após a aprovação da pesquisa pelo comitê de ética, foi realizada a divulgação da pesquisa por meio de cartazes. Estes foram enviados por e-mail para psicólogos acupunturistas formados no Instituto de Psicologia e Acupuntura - Espaço Consciência, com o objetivo de recrutar profissionais habilitados a realizar o tratamento de acupuntura proposto na pesquisa. Cartazes também foram fixados em locais públicos da cidade de São Paulo, convidando o público em geral a participar da pesquisa. Os voluntários que aceitaram participar assinaram o Termo de Consentimento Livre e Esclarecido. Logo em seguida, responderam ao Inventário de Sintomas de Stress de Lipp (ISSL). Na ocasião, considerando os sintomas 
que assinalaram no ISSL, definiram, com a pesquisadora, um sintoma principal de stress a ser tratado que foi denominado queixa. A seguir, foi aplicada a Escala Analógica Visual (EAV) com referência ao sintoma escolhido como queixa, e, por fim, os participantes preencheram o questionário utilizado. Essas avaliações constituíram a fase pré-tratamento.

Além da queixa (sintoma principal do stress), os outros itens assinalados no ISSL e as outras queixas relatadas pelos participantes também foram considerados para o tratamento da acupuntura, apesar de não serem avaliadas pela Escala Analógica Visual.

Assim, os participantes que atingiram os critérios de inclusão iniciaram o tratamento de acupuntura.

\section{Tratamento de acupuntura}

Os participantes submetidos ao ISSL e à EAV, e triados de acordo com os critérios de inclusão da pesquisa, foram reavaliados segundo a medicina tradicional chinesa - acupuntura, buscando-se identificar os desequilíbrios energéticos, de acordo com os sinais e os sintomas apresentados pelo participante.

Assim sendo, para cada participante, definiu-se um princípio terapêutico e um procedimento terapêutico:

1 - Princípio terapêutico

Para o princípio terapêutico, foram

propostos dois modelos distintos:

\section{Fases I, II e III, segundo a MTC - acupuntura} (reagrupamento dos sinais e sintomas).

De acordo com a sintomatologia do participante, distinguiram-se três grupos: Fase I, correspondente à situação de sofrimento inicial; Fase II, com sofrimento intermediário, e Fase III, com sofrimento avançado e perda de recursos para respostas efetivas ao autoequilíbrio.
Análise do modelo pentagonal - Escola dos Cinco Elementos. Procedeu-se à verificação dos sinais e sintomas dos participantes e à avaliação dos microssistemas (pulso, língua e orelha).

\section{2 - Procedimento terapêutico}

Quanto ao procedimento terapêutico, foi utilizada a acupuntura sistêmica, com acupuntura no corpo todo, como se segue:

1) escolheu-se um vaso maravilhoso, de acordo com o desequilíbrio de cada participante. Os vasos maravilhosos, em número de oito, começam e terminam a partir de pontos dos meridianos principais (Cordeiro \& Cordeiro, 2001).

Para Tymowski, Guillaume e Fiévet-Izard (1986), o vaso maravilhoso é um sistema de autorregulação que banha uma região, nela interferindo. Ao levar a energia ancestral a uma determinada região do corpo através do vaso maravilhoso, se um meridiano estiver em excesso, a energia ancestral que banha essa região levará embora esse excesso e, ao contrário, o nutrirá, caso esteja ele insuficiente.

2) equilibrou-se o pentagrama (Escola dos Cinco Elementos), de acordo com o desequilíbrio de cada participante;

3) escolheram-se pontos de ação fisiológica ou sintomáticos da fase em que se encontrava o participante: Fase I, II, ou III.

Foi também adotada a acupuntura auricular como procedimento terapêutico. Colocaramse algumas sementes de mostarda em alguns pontos da orelha, de acordo com o desequilíbrio de cada participante, o que é um procedimento usual na acupuntura (Nogier \& Boucinhas, 1997).

O tratamento foi realizado em 10 sessões individuais, com frequência semanal e duração 
de aproximadamente 50 minutos, incluindo desde o preenchimento do Questionário de Avaliação e Procedimentos para o Tratamento de Acupuntura até a aplicação das agulhas e outros procedimentos da MTC. Também foi utilizado durante o tratamento de acupuntura a folha de registro, como já mencionado anteriormente.

Vale ressaltar que, para este estudo, definiu-se para o tratamento de acupuntura 10 sessões, embora, em geral, tal número possa ser diminuído ou expandido de acordo com a necessidade de cada paciente.

Após as 10 sessões, os participantes imediatamente foram reavaliados e submetidos aos mesmos instrumentos de avaliação psicológica (EAV e ISSL) utilizados na fase pré-tratamento.

\section{Resultados}

No que diz respeito à queixa (sintoma principal do ISSL), foi predominante o cansaço constante, com $30 \%$, seguidos pelos participantes que se queixavam de pensar constantemente em um só assunto e ansiedade/angústia diária, com 15\% em cada queixa. A média do tempo da queixa da amostra foi de 36,7 meses, sendo 1 o menor tempo, e 120, o maior.

\section{Análise comparativa entre as avaliações antes e após o tratamento de acupuntura}

Pelos resultados, verifica-se diferença significativa entre as avaliações (ISSL e EAV) antes e após o tratamento de acupuntura, para as seguintes variáveis: presença de stress (Figura 2), fases de stress (Figura 3), predominância de sintomas (Figura 4) e intensidade da queixa (Figura 5).

Na Figura 2, são ilustrados os resultados obtidos por meio do ISSL antes e após o tratamento de acupuntura desta amostra, enfatizando a presença ou não de stress que, de acordo com o Teste de McNemar, obteve grau de significância $p<0,001$. Pode-se observar que, com o tratamento de acupuntura, houve uma redução de $75 \%$ da presença de stress na amostra estudada.

Quanto às fases de stress, como mostra a Figura 3, os resultados obtidos por meio do ISSL, antes e após o tratamento de acupuntura dessa amostra, de acordo com o teste de simetria, apontaram grau de significância $p=0,006$. Assim, verifica-se que, com o tratamento de acupuntura, houve uma redução do nível de stress dos participantes. Antes do tratamento, os participantes, todos com stress, encontravam-se nas fases de resistência $(60 \%)$, de quase-exaustão (35\%) e de alerta (5\%), e, após o tratamento, os participantes com stress, reduzidos a $25 \%$, passaram a se encontrar somente nas duas primeiras fases de stress: resistência (20\%) e alerta $(5 \%)$.

No que diz respeito à predominância de sintomas, como é ilustrado na Figura 4, os resultados obtidos de acordo com o ISSL, antes e após o tratamento de acupuntura dessa amostra, apontaram grau de significância $p=0,02$, por meio do teste de simetria. Antes do tratamento de acupuntura, pode-se observar que houve predominância de sintomas psicológicos (65\%), físicos (30\%) e de ambos (5\%). Após o tratamento, dos $25 \%$ dos participantes com stress, houve predominância de sintomas psicológicos (20\%) e físicos (5\%).

Na Figura 5, são ilustrados os resultados obtidos por meio da EAV, antes e após o tratamento de acupuntura dessa amostra, enfatizando a intensidade da queixa que, de acordo com o Teste de Wilcoxon, obteve grau de significância $p<0,001$. Pode-se observar que, com o tratamento de acupuntura, houve uma redução na média da intensidade da queixa de 8,1 para 3,2 na amostra estudada. 


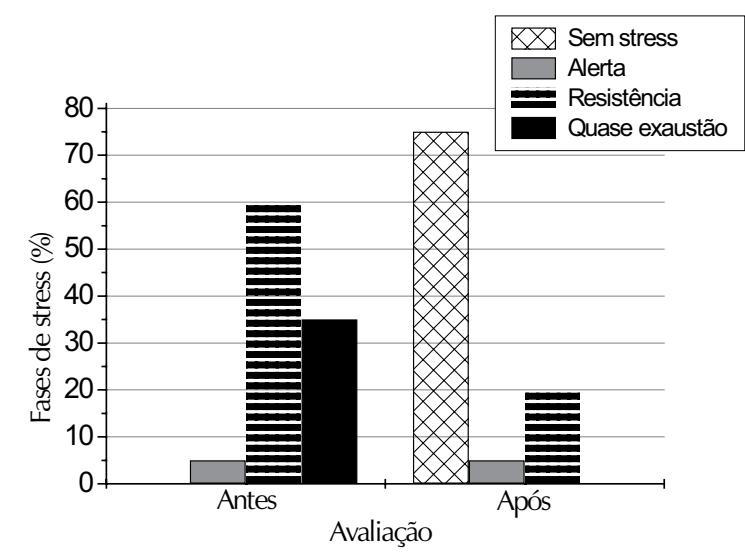

Figura 2 . Comparação da presença de stress antes e após o tratamento de acupuntura Fonte: Dados da pesquisa

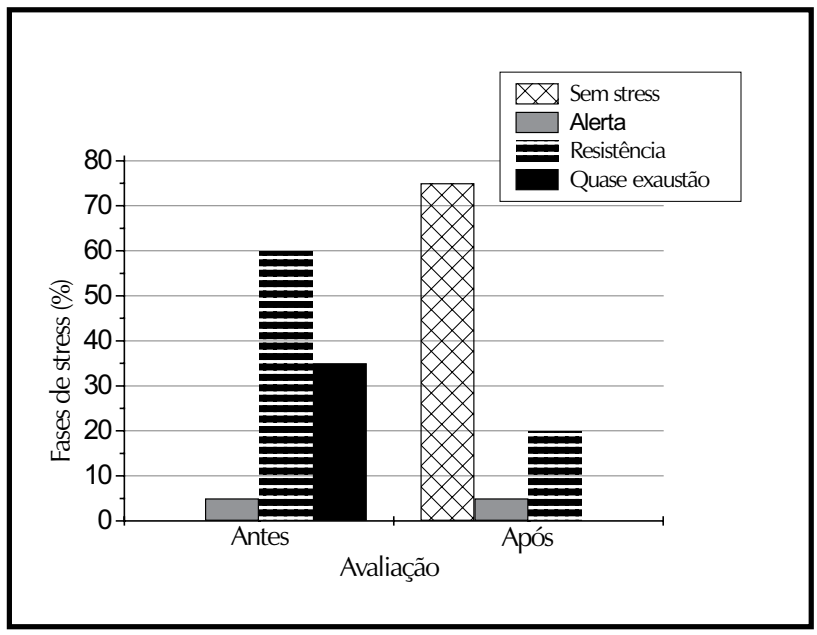

Figura 3. Comparação das fases de stress antes e após o tratamento de acupuntura Fonte: Dados da pesquisa

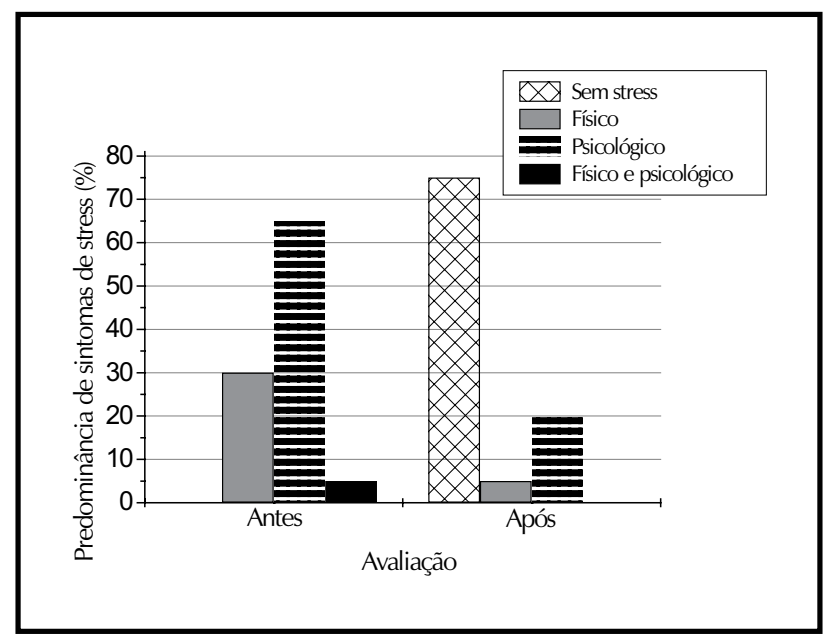

Figura 4. Comparação da predominância de sintomas antes e após o tratamento de acupuntura Fonte: Dados da pesquisa 


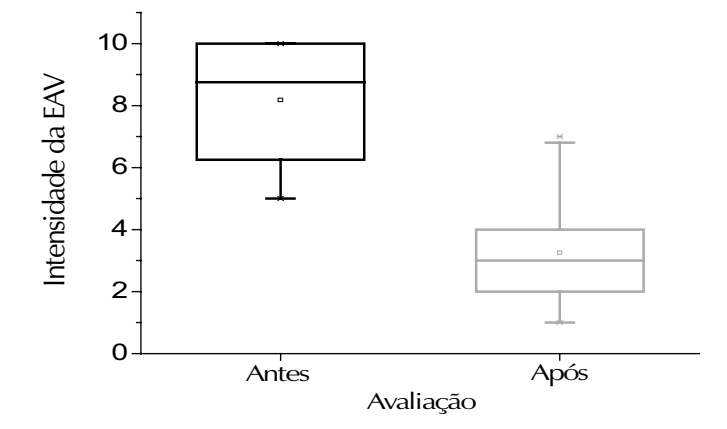

Figura 5. Comparação da intensidade da queixa antes e após o tratamento de acupuntura Fonte: Dados da pesquisa

\section{Discussão}

Com relação aos dados sociodemográficos, a amostra foi composta por 20 participantes, 15 mulheres e 5 homens, ou seja, 75\% da amostra foi de mulheres, o que está de acordo com outros trabalhos, que também mostram maior procura do sexo feminino por tratamentos em geral (Figueiredo, 2005; Pinheiro, Viacava, Travassos, \& Brito (2002).

A faixa etária de 21 a 65 anos foi um critério de inclusão da amostra, já que a demanda pelo tratamento de acupuntura é maior nessa faixa etária, de acordo com o levantamento estatístico dos atendimentos ambulatoriais gratuitos oferecidos no Instituto de Psicologia e Acupuntura da cidade de São Paulo, conforme estudo realizado por esta pesquisadora, antes de iniciar a presente pesquisa. A média da idade desta pesquisa foi de 43,5 anos, sendo 27 a menor idade e 65 a maior. Considerando a ampla faixa etária utilizada nesta pesquisa (27 a 65 anos), seria interessante que trabalhos futuros analisassem os efeitos do tratamento utilizado por idade, dividindo a faixa etária em períodos mais curtos.

No que se refere à escolaridade, predominaram os participantes com ensino superior completo (65\%). No estudo de Lima (2007), o resultado foi semelhante, pois, ao se avaliarem os entrevistados por nível de escolaridade, destacou-se grande procura da acupuntura por pessoas com boa instrução, sugerindo que parte significativa dos participantes no presente estudo possui bom nível socioeconômico e cultural.

No contexto profissional, a amostra foi bastante variada, com predominância das atividades do lar, jornalista, aposentado e psicólogo. Isso mostra, mais uma vez, a presença de pessoas teoricamente instruídas e/ou com conhecimento na área de saúde, em busca de uma terapia complementar como forma de tratamento.

Quanto à queixa (sintoma principal do ISSL), foi bastante variada, com predominância de participantes que apresentavam cansaço constante (30\%), seguidos por outros que se queixavam de pensar constantemente em um só assunto (15\%) ou de apresentar ansiedade/angústia diária 
(15\%). Especificamente em relação aos sintomas mais assinalados no ISSL antes do tratamento de acupuntura, foram: sensação de desgaste físico constante (95\%), cansaço constante (90\%), tensão muscular (85\%) e ansiedade/angústia diária (85\%). Em uma pesquisa realizada com estudantes adultos jovens (Calais, Andrade, \& Lipp, 2003), os sintomas mais mencionados pelas mulheres foram: a sensibilidade emotiva exagerada, seguida de irritabilidade excessiva, sensação de desgaste físico constante e cansaço constante. Já para os homens, o sintoma mais presente foi o pensamento constante sobre um só assunto, seguido da sensação de desgaste físico constante e problemas com a memória. Desse modo, tanto homens como mulheres mostraram que a sensação de desgaste físico constante é muito frequente na pessoa estressada, assim como foi encontrado na presente pesquisa. Adicionalmente, em outro estudo realizado com juízes (Lipp \& Tanganelli, 2002), os resultados também foram semelhantes, sendo mencionados como sintomas mais frequentes: sensação de desgaste físico constante ( $71 \%)$, tensão muscular $(60 \%)$ e irritabilidade excessiva (52\%).

A média do tempo da queixa (sintoma principal do ISSL) foi de 36,7 meses, sendo o menor de 1 mês e o maior de 120 meses. A maioria dos participantes relatou já ter procurado outros tipos de tratamento antes da acupuntura, porém os resultados não foram satisfatórios e, por isso, havia um tempo grande de permanência da queixa. Esse dado corrobora a pesquisa de Lima (2007), que observou que apenas 9\% dos entrevistados não fizeram outros tipos de tratamentos anteriores. A grande maioria utilizou-se de tratamentos farmacológicos, principalmente com uso de anti-inflamatórios não hormonais, relaxantes musculares e medicamentos não especificados. A fisioterapia foi uma forma de tratamento também muito procurada, na maioria das vezes acompanhada do uso de medicamentos.
Sendo assim, especula-se que a busca por abordagens complementares, entre elas a acupuntura, deve-se, principalmente, a uma insatisfação com a Medicina convencional. Antes do tratamento de acupuntura, todos os participantes apresentavam stress de acordo com o ISSL, já que esse era um dos critérios de inclusão da amostra. Dentre os participantes, verificou-se que a maioria se encontrava na fase de resistência (60\%), seguida da fase de quase-exaustão (35\%), alerta (5\%) e nenhum na fase de exaustão do stress. Isso indica que os participantes apresentavam sintomas relativos ao desgaste físico generalizado, caracterizado por sensação de perda de energia e cansaço físico. De fato, essa concentração é provável, como demonstrou Lipp (2004) nas pesquisas de validação do Inventário de Sintomas de Stress para adultos (ISSL), pois significa que o stress desses participantes é de grande duração ou de grande intensidade e, eventualmente, alguns dos sintomas da fase de alerta, especialmente aqueles relacionados à motivação, desaparecem, predominando os sintomas de desgaste e sensação de cansaço.

No que se refere à predominância de sintomas, a maioria apresentou sintomas psicológicos (65\%), seguidos pelos sintomas físicos (30\%), e apenas um participante apresentou sintomas físicos e psicológicos (5\%). Pode-se observar que, em uma pesquisa realizada com estudantes adultos jovens (Calais, Andrade, \& Lipp, 2003), os resultados foram semelhantes, sendo que a predominância, nos estudantes, foi de sintomatologia psicológica $(55,7 \%)$, seguida de sintomas físicos $(32,4 \%)$ e de ambos (11,9\%). Em outra pesquisa realizada com técnicos da área da saúde (Malagris \& Fiorito, 2006), os resultados também foram semelhantes, sendo que, dentre os estressados, houve maior incidência de manifestação de sintomas psicológicos (69\%), seguindo-se os físicos (27\%) e os dois concomitantemente (4\%). 
Os participantes também foram avaliados antes do tratamento de acupuntura pela EAV, que verificou a intensidade da queixa (sintoma principal do ISSL), obtendo-se a média de 8,1 , sendo que 5 foi a menor nota e 10 a maior. Isso mostra que os participantes apresentavam um incômodo significativo em relação à intensidade da queixa antes do tratamento de acupuntura.

Após as avaliações do ISSL e da EAV, os participantes foram reavaliados segundo a medicina tradicional chinesa - acupuntura, buscando-se identificar os desequilíbrios energéticos de acordo com os sinais e os sintomas apresentados. A pesquisadora, juntamente ao professor Delvo Ferraz da Silva, propôs uma intervenção da medicina tradicional chinesa - acupuntura como forma de tratamento na redução da sintomatologia do stress.

Após a intervenção de acupuntura, verificouse nessa amostra uma redução dos sintomas de stress e da intensidade da queixa (sintoma principal do ISSL) dos participantes. Pessoas que, antes do tratamento, encontravam-se nas fases de alerta, resistência e de quaseexaustão, após o tratamento, em sua maioria (75\%), não mais apresentavam stress, ou regrediram às fases iniciais de alerta (5\%) e resistência (20\%). No que se refere à predominância de sintomas, $75 \%$ não os apresentaram, ou seja, encontravam-se sem stress, em $20 \%$, predominaram os sintomas psicológicos e, em 5\%, os sintomas físicos. Pode-se observar que a média da intensidade da queixa (sintoma principal do ISSL), após o tratamento de acupuntura, passou de 8,1 para 3,2 na amostra estudada. Sendo assim, pelos resultados dessa amostra, verifica-se diferença significativa entre as avaliações, tanto no ISSL como no EAV, antes e após o tratamento de acupuntura, para as seguintes variáveis: presença de stress, fases de stress, predominância de sintomas e intensidade da queixa.
Há que se considerar, no entanto, que a avaliação pós-tratamento foi realizada imediatamente após essa fase, não se tendo ainda dados quanto à manutenção dos resultados da acupuntura na redução dos sintomas de stress.

No estudo de Pavão (2008), a intervenção de acupuntura foi capaz de reduzir significativamente os sintomas de stress, ansiedade e depressão de 12 adultos jovens (entre 23 e 38 anos) e de 12 idosos (entre 60 e 81 anos), além de aumentar a proliferação linfocitária, que contribuiu para o aumento da imunidade do grupo estudado.

Desse modo, as pesquisas sobre os efeitos da acupuntura têm demonstrado que, estimulando os pontos indicados, ocorre uma reação que se dá através da liberação de peptídios opioides no sistema nervoso, provocando efeito analgésico, conforme apontam Ernest e White (2001).

White (2000) discorre sobre a importância de os psicólogos conhecerem e estudarem cientificamente as abordagens complementares, como a acupuntura, pois estatísticas mostram que, em 1997, 42\% da população americana utilizou tratamento não convencional, gastando cerca de $\$ 21.2$ bilhões de dólares com essas práticas, conforme apontam Eisenberg, Davis, Ettner, Appel, Wilkey, Van Rompay \& Kessler (1998). Dentre as patologias mais suscetíveis a tais terapêuticas, encontram-se a dor nas costas, a dor de cabeça, a ansiedade e a depressão - estas últimas, patologias prioritariamente tratadas por psicólogos.

Pode-se, assim, ressaltar a pertinência de novos estudos que ampliem o conhecimento sobre a efetividade da técnica junto à abordagem psicológica. 


\section{Conclusão}

A acupuntura é uma forma de terapia milenar, baseada em princípios próprios da MTC, cujos conceitos de saúde e etiopatogenia das doenças e cuja forma de diagnosticar e direcionar o tratamento são permeados por conhecimentos distintos daqueles presentes na Medicina ocidental.

Conclui-se, a partir dos resultados da presente pesquisa, que o tratamento de acupuntura foi útil na redução da sintomatologia do stress dessa amostra, considerando-se seus efeitos imediatos.

Os resultados obtidos na amostra demonstraram diferença significativa entre as avaliações (ISSL e EAV) antes e após o tratamento de acupuntura para as seguintes variáveis: presença de stress, fases de stress, predominância de sintomas e intensidade da queixa, porém ainda não se possuem dados sobre a manutenção desses ganhos. Os dados corroboram os resultados da literatura científica acerca de pesquisas realizadas, em que a intervenção de acupuntura foi capaz de reduzir significativamente os sintomas.

É importante assinalar que este estudo foi realizado com uma amostra reduzida e com uma faixa etária ampla e que, portanto, as conclusões aqui apresentadas devem ser consideradas de forma cuidadosa, evitandose generalizações precipitadas. Sugere-se a realização de estudos futuros, com amostra maior, com uma faixa etária mais homogênea, e também a inclusão de um grupo controle, como, por exemplo, indivíduos tratados com pontos falsos inseridos na pele, o que permitiria melhor controle sobre o efeito placebo inerente a qualquer intervenção.

Pretende-se que esta pesquisa amplie os conhecimentos teórico-científicos sobre o tema stress e acupuntura, uma vez que existem poucos estudos sistematizados nessa área. Espera-se, enfim, que o estudo contribua para a elaboração de terapêuticas para o controle do stress como forma de garantir a saúde e o bem-estar do ser humano.

\section{Marília Conceição da Silva Doria}

Mestre em Psicologia pela Pontifícia Universidade Católica de Campinas, colaboradora de pesquisa no Laboratório de Estudos Psicofisiológicos do Stress - PUC-Campinas, São Paulo, SP -Brasil.

E-mail: mari_doria@yahoo.com.br

\section{Delvo Ferraz da Silva}

Especialização em acupuntura pela Sociedade Brasileira de Acupuntura e Psicologia - SOBRAPA, São Paulo, SP - Brasil.

E-mail: acupuntura@psicologiaeacupuntura.com.br

\section{Marilda Emmanuel Novaes Lipp}

Doutorado em Ph D em Psicologia Experimental e Clínica - George Washington University e pós doutorado realizado no National Institute of Health, Maryland, EUA. É professora titular da Pontifícia Universidade Católica de Campinas, São Paulo, SP - Brasil.

E-mail: mlipp@uol.com.br

\section{Endereço para envio de correspondência:}

Rua Santa Sardelli Ribeiro, 87- Solário da Mantiqueira São João da Boa Vista, São Paulo - SP. CEP: 13875-428

Recebido 1/3/2010, 1ㅍ- Reformulação 4/9/2011, Aprovado 15/10/2011. 


\section{Referências}

Breves, R. (2001). Acupuntura tradicional chinesa. São Paulo: Robe.

Calais, S.L., Andrade, L.M.B., \& Lipp, M.E.N. (2003). Diferenças de sexo e escolaridade na manifestação do stress em adultos jovens. Psicologia: Reflexão \& Crítica, 16(2), 257-263.

Campiglia, H. (2004). Psique e medicina tradicional chinesa. São Paulo: Roca.

Cordeiro, A. T. (1994). Acupuntura. São Paulo: Ensaio.

Cordeiro, A. T, \& Cordeiro, R. C. (2001). Acupuntura: elementos básicos (3a ed.). São Paulo: Polis.

Eisenberg, D.M.; Davis, R.B.; Ettner, S.L.; Appel, S.; Wilkey, S.; Van Rompay, M.; Kessler, R.C. (1998). Trends in Alternative Medicine use in the United States, 1990-1997. Journal of the American Medical Association, 280, 569-1575.

Ernest, E.; White, A. (2001). Acupuntura: Uma avaliação científica. São Paulo: Manole.

Faubert, G., \& Crepon, P. A. (1990). Cronobiologia chinesa. São Paulo: Ibrasa.

Holmes, T. H., \& Rahe, R. K. (1967). The social readjustment ranting scale. Journal Psychossomatic, 4, 189 -194.

Kidson, R. (2006). Acupuntura para todos: o que esperar dessa técnica milenar e como obter melhores resultados. Rio de Janeiro: Nova Era.

Lima, J.H.C. (2007). Estudo da demanda de Tratamento por Acupuntura no hospital universitário da Universidade Federal de Santa Catarina. Monografia de Graduação, Faculdade de Medicina, Universidade Federal de Santa Catarina, Florianópolis.

Lipp, M. E. N. (1984). Stress e suas implicações. Estudos de Psicologia, 1(3-4), 5-19.

Lipp, M. E. N. (1996). Pesquisas sobre stress no Brasil: saúde, ocupações e grupos de risco. São Paulo: Papirus.

Lipp, M. E. N. (2000). Manual do inventário de sintomas de stress para adultos. São Paulo: Casa do Psicólogo.

Lipp, M. E. N., \& Malagris, L. E. N. (1995). Manejo do estresse. In B. Range (Org.), Psicoterapia comportamental e cognitiva: pesquisa, prática, aplicação e problemas (pp. 279-292). Campinas, SP: Editorial Psy II.
Lipp, M. E. N., \& Rocha, J. C. (1996). Stress, hipertensão e qualidade de vida (2a ed.). Campinas, SP: Papirus.

Maciocia, G. (1996). Os fundamentos da medicina chinesa. São Paulo: Roca.

Malagris, L.E.N. \& Fiorito, A.C.C. (2006). Avaliação do nível de stress de técnicos da área de saúde. Estudos de Psicologia, 23(4). Recuperado em 23 de janeiro de 2009 de http://www. scielo.br/scielo.php?pid=S0103166X2006000400007\&scrip $\mathrm{t}=\mathrm{sci}$ arttext \&tlng=pt

Nogier, R. \& Boucinhas, J.C. (1997). Prática fácil de auriculoterapia e auriculomedicina. São Paulo: Ícone.

Pavão, T.S. (2008). Efeitos de uma intervenção de acupuntura sobre sintomas psicológicos e imunidade celular de adultos jovens e idosos saudáveis. Dissertação de Mestrado, Programa de Pós-Graduação em Gerontologia Biomédica, Pontifícia Universidade do Rio Grande do sul, Porto Alegre.

Pinheiro R.S.; Viacava F.; Travassos C. \& Brito A.S. (2002). Gênero, morbidade, acesso e utilização de serviços de saúde no Brasil. Ciência Saúde Coletiva, 7, 687-707.

Requena, Y. (1990). Acupuntura e psicologia. São Paulo: Andrei.

Selye, H. (1965). Stress - a tensão da vida (2a ed.) F. Bronco, trad.). São Paulo: Ibrasa.

Spielberger, C. (1979). Understanding stress and anxiety. New York: Harper \& Row Publishers.

Tymowski, G., \& Fiévet-Izard, M. (1986). A acupuntura Rio de Janeiro: Jorge Zahar Editora. (Coleção Cultura Comtemporânea)

Wen, T. S. (1989). Acupuntura clássica chinesa (2a ed.). São Paulo: Cultrix.

White, K. P. (2000). Psychology and Complementary and Alternative Medicine. Professional Psychology: Research and Practice, 31(6), 671-681.

Yamamura, Y. (1993). Acupuntura tradicional: a arte de inserir. São Paulo: Roca. 